A N N A L E S Annales de Bretagne et des Pays de l'Ouest

BRETAGNE PAYS DE LOUEST

Anjou. Maine. Poitou-Charente. Touraine

125-1 | 2018

Tolérance et intolérance des religions en Europe, $\mathrm{XVI}$ -

XVIII siècle

\title{
Une tolérance à géométrie variable : catholiques et protestants, chrétiens et juifs, pouvoir royal et francisation
}

Metz au XVII ${ }^{\mathrm{e}}$ siècle

Varying degrees of toleration: Catholics and Protestants, Christians and Jews, royal power and Frenchification. $17^{\text {th }}$ century Metz

Julien Léonard

\section{(2) OpenEdition}

Journals

Édition électronique

URL : http://journals.openedition.org/abpo/3777

DOI : $10.4000 /$ abpo.3777

ISBN : 978-2-7535-7536-3

ISSN : 2108-6443

Éditeur

Presses universitaires de Rennes

Édition imprimée

Date de publication : 30 mars 2018

Pagination : 97-109

ISBN : 978-2-7535-7491-5

ISSN : 0399-0826

Référence électronique

Julien Léonard, « Une tolérance à géométrie variable : catholiques et protestants, chrétiens et juifs, pouvoir royal et francisation ", Annales de Bretagne et des Pays de l'Ouest [En ligne], 125-1 | 2018, mis en ligne le 30 mars 2020, consulté le 05 janvier 2021. URL : http://journals.openedition.org/abpo/3777 ; DOI : https://doi.org/10.4000/abpo.3777 


\title{
Une tolérance à géométrie variable : catholiques et protestants, chrétiens et juifs, pouvoir royal et francisation
}

\author{
Metz au XVII ${ }^{\mathrm{e}}$ siècle
}

\author{
Julien LÉONARD \\ Maître de conférences en histoire moderne \\ université de Lorraine - CRULH (EA 3945), Nancy
}

En regard des situations bien étudiées de coexistence confessionnelle au sein du royaume de France aux XVI ${ }^{\mathrm{e}}$ et $\mathrm{XVII}^{\mathrm{e}}$ siècles, mais aussi dans les zones impériales frontalières, le cas de la ville de Metz (20000 habitants environ) constitue une originalité qu'il ne faut certes pas exagérer, mais dont il faut analyser les causes. En effet, elle bénéficie d'un régime de coexistence entre catholiques et réformés proche de celui de l'édit de Nantes. La communauté réformée y est puissante, nombreuse et bien établie $^{1}$. Mais surtout, et c'est là que réside la principale originalité, la ville abrite une communauté juive ${ }^{2}$. Ces juifs de Metz sont des privilégiés, ils sont exempts de l'application de l'édit d'expulsion de 1394 et de son renouvellement en 1615. Ils ont été officiellement autorisés à s'installer au milieu des années 1560 par les agents du roi de France, notamment pour des raisons financières et économiques.

La ville n'est en théorie qu'occupée par la France, depuis 1552, et elle reste juridiquement une ville libre d'Empire, ce qui lui donne un statut à part $^{3}$. L'annexion officielle de Metz à la France par les traités de Westphalie

1. Tribout de Morembert, Henri, La Réforme à Metz, Nancy, PUN, 2 vol., 1969-1971;

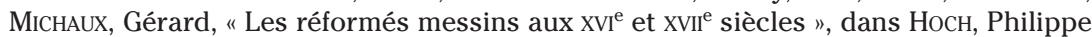
(éd.), Huguenots. De la Moselle à Berlin, les chemins de l'exil, Metz, Éditions Serpenoise, 2006, p. 17-46.

2. ClÉment, Roger, La condition des Juifs de Metz sous l'Ancien Régime, Paris, Henri Jouve, 1903; MEYER, Pierre-André, La communauté juive de Metz au XVIII siècle : histoire et démographie, Nancy/Metz, PUN/Éditions Serpenoise, 1993.

3. ZELLER, Gaston, La réunion de Metz à la France (1552-1648), Paris, Les Belles Lettres, 2 vol., 1926; PETRY, Christine, "Faire des sujets du roi ». Rechtspolitik in Metz, Toul und Verdun unter französischer Herrschaft (1552-1648), Munich, Oldenbourg, 2006. 
en 1648 - situation déjà largement intégrée par les habitants et même institutionnalisée lors des décennies précédentes, notamment par l'installation d'un parlement en 1633 - ne change pas immédiatement les choses. La ville continue à bénéficier de certaines souplesses dans sa relation avec la monarchie : jusqu'au début des années 1660 au moins, les députations de l'assemblée des Trois-Ordres de Metz peuvent accéder assez librement au souverain et lui présenter des remontrances ou des doléances qui sembleraient bien hardies pour d'autres.

C'est la situation géopolitique de Metz qui explique une situation confessionnelle qui relève d'une prise en compte pragmatique des intérêts de la monarchie sur sa frontière est, toujours fragile et mouvante. Devant tenir compte de l'héritage du XvI ${ }^{\mathrm{e}}$ siècle, le pouvoir royal agit avec prudence. Toutefois, le XVII ${ }^{\mathrm{e}}$ siècle voit évoluer les situations de tolérance civile que connaissent les deux communautés minoritaires. Alors que les réformés tendent à rentrer dans le rang et à connaître, de plus en plus, le sort commun de leurs coreligionnaires du royaume, les juifs voient leur particularisme confirmé, malgré l'hostilité de la population chrétienne. Ces évolutions, qui ne sont pas linéaires, s'expliquent par la francisation de la ville : cette hypothèse est au cœur de la thèse de l'historienne Patricia Behre Miskimin, qui voit dans le triconfessionnalisme messin un moyen de développement de l'État royal à Metz ${ }^{4}$, ainsi que, très récemment, d'un article de Stephan Laux, qui interprète le maintien des privilèges des juifs comme le moyen de limiter les effets négatifs des vexations faites aux protestants, dans le but de trouver un Ersatzbürgertum ${ }^{5}$.

La tolérance décrira ici non un concept philosophique, mais l'attitude pragmatique du pouvoir royal et de ses représentants, dont le but est surtout politique. Ils acceptent jusqu'en 1685 la présence sur le territoire de la ville de Metz de communautés minoritaires, dans le but de renforcer l'emprise française. Cela ne préjuge en rien de certaines situations de coexistence pacifique dans la vie quotidienne, faite d'accommodements parfois entre les Messins, en tout cas entre les chrétiens, ni de l'attitude a contrario franchement hostile des autorités ecclésiastiques de part et d'autre, cette intolérance institutionnelle des " hommes de Dieu " et des Églises, et notamment de la catholique.

\section{Une ville, trois cultes : un cas exceptionnel, fruit d'un héritage}

Lorsque les Français entrent à Metz en avril 1552, ils trouvent une situation confessionnelle complexe, qui va les forcer à composer pour ne pas s'aliéner les populations locales et les princes protestants allemands qui

4. Behre Miskimin, Patricia, One King, One Law, Three Faiths. Religion and the Rise of Absolutism in Seventeenth-Century Metz, Westport/Londres, Greenwood Press, 2002.

5. LAux, Stephan, "Ersatzbürgertum" in the Wake of Confessionalization: Jews, Protestants, and French Royal Administration in Metz (16 $6^{\text {th }} 17^{\text {th }}$ Century) ", Frankfurter Judaistische Beiträge, t. 40, 2015, p. 285-307. 
ont permis l'occupation de la ville par le traité de Chambord. Certes, les réformés vivent de façon clandestine, mais ils sont influents en ville, y compris parmi les élites dirigeantes, et ils sont proches des protestants suisses et strasbourgeois depuis le passage dans la région de Guillaume Farel en 1542-1543. C'est à la fin des années 1550 et au début des années 1560 que l'Église réformée de Metz se constitue, selon des modèles proches de ceux de France et de Genève ${ }^{6}$, avec un consistoire rassemblant des pasteurs, des anciens et des diacres. Le culte est toléré intra muros en 1561, dans un endroit cependant excentré. L'apogée du protestantisme messin peut être daté de 1565, avec sans doute la moitié de la population adhérant au calvinisme, et le dernier voyage de Farel. Mais la période des guerres de Religion rend la vie des protestants instable, ballottés qu'ils sont entre périodes d'interdiction, notamment suite à la destruction du temple (1569-1576, puis 1577-1592), et courtes périodes de tolérance (1561-1569, 1576-1577). Dans les faits, ils ne perdent toutefois pas réellement de terrain. Ils peuvent trouver des expédients pour pallier les vexations, et exercer de façon clandestine ou dans des lieux isolés, grâce à des gouverneurs qui ferment les yeux, y compris le duc d'Épernon. Entre 1592 et 1597, cette situation d'entredeux semble même s'installer, avec un culte certes en plein air, mais près des remparts et quasiment au grand jour, avec l'accord des autorités. Le pouvoir royal sait donc moduler ses attentes et accepter de tolérer de fait les réformés, y compris quand ils sont interdits légalement. En 1597 enfin, des mesures sont prises par Henri IV et ses relais en ville pour autoriser à nouveau le culte en ville, avec un nouveau temple qui n'est achevé que dans les années 1610, et un statut raffermi par son inclusion aux articles particuliers de l'édit de Nantes ${ }^{7}$. Pourtant, ils n'ont pas le droit d'user de leur lieu de culte construit en 1576 en plein cour de la ville, car les catholiques s'efforcent visiblement de les en empêcher et de les maintenir dans un statut de religion simplement tolérée, comme dans les années 1560 : un brevet de l'édit de Nantes lui-même interdit le culte dans l'ancien temple ${ }^{8}$. Socialement et politiquement, les réformés sont pourtant très puissants au cours des premières décennies du XVII ${ }^{\mathrm{e}}$ siècle, même s'ils ne représentent déjà plus qu'un tiers de la population en 1635, et les élites n'hésitent pas à s'engager dans l'Église. Encadrés par des pasteurs réputés, ils maintiennent une piété très vivante et jouissent d'une certaine tranquillité, en tout cas en comparaison avec leurs coreligionnaires du royaume.

6. LÉONARD, Julien, «Un protestantisme français? Metz et le Pays messin sous protection royale (1552-1685) ", dans JALABERT, Laurent et LÉONARD, Julien (dir.), Les protestantismes en Lorraine (XVI-XXI siècles), Villeneuve-d'Ascq, Presses universitaires du Septentrion, 2018, à paraître.

7. BARBICHE, Bernard et al. (éd.), L'édit de Nantes et ses antécédents (1562-1598) [http:// elec.enc.sorbonne.fr/editsdepacification/edit_13], consulté le 25 octobre 2016, art. 9 des particuliers.

8. Ibid. [http://elec.enc.sorbonne.fr/editsdepacification/edit_15], consulté le 25 octobre 2016, art. 9 du brevet des garnisons. 
Le régime de tolérance civile des juifs à Metz est radicalement différent de celui qui a été accordé aux protestants, car leur installation répond à une logique inverse. Alors que les autorités françaises doivent accepter la présence des réformés, antérieure à leur arrivée, ce sont elles qui, au milieu des années 1560, autorisent des ménages juifs en nombre très limité (trois ou quatre ménages) à s'installer, pour des raisons purement contingentes et, pense-t-on alors, limitées dans le temps. Même s'ils étaient présents depuis longtemps dans un espace lorrain politiquement très morcelé, les juifs étaient absents de la ville depuis le début du XIII ${ }^{\mathrm{e}}$ siècle sans doute, et c'est donc un choc pour la population chrétienne de Metz, immédiatement hostile ${ }^{9}$. Dès 1565-1566, des délibérations du Magistrat tentent d'endiguer cette installation, mais en 1567, le gouverneur français, le maréchal de Vieilleville, la valide et place les juifs sous sa protection et celle du roi, à la condition (toute théorique) qu'ils aillent entendre un sermon catholique par mois à la cathédrale ${ }^{10}$. L'attention de la monarchie à l'égard des juifs semble se confirmer tout au long de la seconde moitié du $\mathrm{XVI}^{\mathrm{e}}$ siècle, à une exception près : le 6 février 1574, le lieutenant général Thévalle les expulse $^{11}$. Mais ils sont rappelés par Henri III dès août $1575^{12}$. Des décisions successives autorisent a posteriori des installations par mariage, audelà des quatre ménages initiaux. En 1595, ils sont suffisamment nombreux pour former une communauté instituée, conformément aux exigences rabbiniques, et mettre en place des institutions ${ }^{13}$. Les juifs se choisissent dès lors un grand rabbin, arbitre généralement venu d'Allemagne ou d'Europe centrale et orientale. Disposant dès cette époque d'un oratoire, ils ont une synagogue et un cimetière dans les années 1610 et peuvent exercer leur culte dans des conditions très favorables.

Les communautés tolérées sont réparties spatialement selon des logiques qui répondent à celles de leur installation et qui ont des conséquences sur la façon de vivre au quotidien les situations de tolérance civile. Les réformés vivent dans toutes les paroisses et voisinent avec les catholiques, les deux populations chrétiennes sont donc mélangées. Le XVII ${ }^{\mathrm{e}}$ siècle voit le recul des mariages "bigarrés ", mais il n'y a pas de réel problème à ce que catholiques et réformés vivent côte à côte. En revanche, les juifs sont clairement confinés. Les premières installations se font sans doute dans la paroisse administrative de Saint-Ferroy, et une décision du

9. MeYer, Pierre-André, La communauté juive..., op. cit., p. 27 ; LÉONARD, Julien, « Le regard des chrétiens sur les juifs à Metz aux XVI ${ }^{\mathrm{e}}$ et XVII ${ }^{\mathrm{e}}$ siècles : voisins ou étrangers? ", dans Bourdieu-Weiss, Catherine (éd.), Les Trois-Évêchés et l'étranger, Metz, crulh, 2014, p. 139-165.

10. Emmery, Jean-Louis Claude (éd.), Recueil des Édits, Déclarations, Lettres-Patentes, et Arrêts du Conseil enregistrés au Parlement de Metz, Metz, Pierre Marchal, 1775, t. 1, p. 198-201.

11. ClÉMENT, Roger, La condition des Juifs de Metz..., op. cit., p. 236-237.

12. Bibliothèque nationale de France (BnF), ms. fr. 3388, fol. 7; Gaston ZELLER, La réunion de Metz à la France..., op. cit., t. 2, p. 133-134.

13. CAHEN, Abraham, Le rabbinat de Metz pendant la période française (1567-1871), Paris, Durlacher, 1886, p. 4-5. 
gouverneur, le duc d'Épernon, confirme cet état de fait en janvier 1614, autorisant même les juifs à devenir propriétaires dans cette zone ${ }^{14}$. Même si sa judaïsation n'est que progressive et si on ne peut parler de ghetto ${ }^{15}$, il existe des marqueurs spatiaux du quartier, et les juifs ne peuvent en sortir certains jours de fêtes chrétiennes, et les autres moments uniquement avec les signes ordinaires en Occident depuis le concile de Latran IV. Avant le XVIII siècle, peu nombreux sont les francophones parmi ces juifs ashkénazes parlant le yiddish ${ }^{16}$, et il est certain qu'ils apparaissent en ville comme une sorte de greffe imposée par le pouvoir royal. Même si le cardinal de Givry, durant son court épiscopat (1608-1612), tente de travailler à leur conversion au catholicisme en leur imposant des sermons spécifiques ${ }^{17}$, il est peu probable qu'ils soient de véritables cibles au début du XVII ${ }^{\mathrm{e}}$ siècle.

Cela ne doit pas faire oublier que le culte le plus important, tant du point de vue spatial que numérique, reste le catholicisme. Avec une quinzaine de paroisses, un chapitre cathédral, des chapitres collégiaux et près de trente maisons régulières, les ecclésiastiques catholiques se comptent par centaines en ville et se renforcent avec l'arrivée de nouvelles installations comme les capucins, les jésuites, les minimes, la propagation de la foi, etc. ${ }^{18}$ Le clergé, hostile aux réformés et aux juifs, exerce un pouvoir important en ville, tant socialement qu'institutionnellement, notamment au sein de structures municipales comme l'assemblée des Trois-Ordres. En revanche, il est diminué par la relation ambiguë de la ville au pouvoir épiscopal, traditionnellement ennemi avant 1552 et confiné dans les terres de l'évêché, mais de retour sur la scène politique. Entre 1612 et 1669, il n'y a pas d'évêque résident. Pourtant, grâce à la présence d'agents zélés sur place, l'alliance entre le clergé catholique et les autorités représentant la monarchie tend à se renforcer $\mathrm{au} \mathrm{XVII}^{\mathrm{e}}$ siècle, et il s'agit d'un facteur explicatif majeur pour comprendre la redéfinition des règles de la tolérance civile, parallèlement au renforcement de l'emprise monarchique sur Metz.

En effet, si la ville semble relativement ouverte jusqu'à la guerre de Trente Ans, cette dernière est synonyme de désordres pour les autorités, mais aussi pour les ecclésiastiques catholiques qui dénoncent avec virulence la tolérance de fait qui s'y est installée, comme le précise l'évêque suffragant Martin Meurisse en 1642 :

14. EmmERY, Jean-Louis Claude (éd.), Recueil des Édits..., op. cit., t. 1, p. 206-207.

15. MEYER, Pierre-André, La communauté juive..., op. cit., p. 48.

16. GOETSCHEL, Roland, "Le monde ashkénaze aux XVI ${ }^{\mathrm{e}}$-XVIII ${ }^{\mathrm{e}}$ siècles : une histoire religieuse ", dans Germa, Antoine, Lellouch, Benjamin et Patlagean, Évelyne (dir.), Les Juifs dans l'histoire, de la naissance du judaïsme au monde contemporain, Seyssel, ChampVallon, 2011, p. 375-384.

17. [Pérusse des Cars, Anne DE, cardinal de Givry (dir.)], Statuta Synodi Dioecesance Metensis Primce, Metz, Fabert, 1610, p. 110.

18. MichauX, Gérard, "Réforme catholique et Contre-Réforme à Metz au XVII siècle ", dans Le Moigne, François-Yves et Michaux, Gérard (éd.), Protestants messins et mosellans (XVIT-XXe siècles), Metz, Éditions Serpenoise/SHAL, 1988, p. 47-70. 
"L'on vit, en mesme temps, l'exercice libre de cinq ou six sectes differentes, dans l'enclos des murailles. Car outre le Sabbath des Juifs, \& les presches ordinaires à la Calvinienne, il y avoit encor trois ou quatre presches à la Lutherienne, en divers endroits de la Ville ${ }^{19}$."

Dans une ville frontière de plus en plus sensible et délicate dans la stratégie de Louis XIII et Richelieu ${ }^{20}$, il faut mettre de l'ordre, tout en restant pragmatique. Ainsi, les deux cultes légalement tolérés connaissent, à partir du second tiers du XVII ${ }^{\mathrm{e}}$ siècle, des destins croisés.

\section{Une tolérance remise en cause : l'érosion des privilèges des réformés}

L'histoire de la remise en cause du régime de tolérance accordé aux réformés à Metz est celle d'une normalisation et, donc, d'une certaine forme de francisation. En effet, même si les protestants de Metz bénéficient jusque dans les années 1680 d'aménagements dans les vexations qui sont faites à leurs coreligionnaires du royaume - ce qu'Élie Benoist prendra d'ailleurs pour un manque de solidarité ${ }^{21}$ - ils connaissent de plus en plus le sort commun, bien connu par l'historiographie ${ }^{22}$.

Au début du XVII ${ }^{\mathrm{e}}$ siècle, l'Église réformée de Metz est indépendante des structures synodales françaises avec lesquelles elle se considère toutefois liée, comme avec Genève ${ }^{23}$. Elle bénéficie d'un régime de tolérance avantageux, plus que celui de France, puisque n'étant pas soumise à la surveillance de commissaires royaux ou encadrée dans la violence verbale contre le " papisme ". Les protestants jouissent également, malgré les attaques catholiques, d'un statut politique particulier, notamment au sein des institutions municipales. C'est ce qui vaut à Metz d'être qualifiée en 1612 par le vicaire général de l'évêché, André Valladier, de la " ville de France la plus gangrenée de ceste pestilente gratelle ${ }^{24}$ ». En novembre 1628 encore, le pouvoir intervient, par la voix du commandant d'alors, Fromigières, pourtant proche des milieux dévots, pour condamner les débordements de ceux qui célébraient avec trop de zèle la victoire des troupes royales à La Rochelle ${ }^{25}$. La liberté de recrutement des pasteurs ou la richesse du consistoire et de la bourse des diacres en faveur des pauvres rendent l'Église de Metz attractive et rayonnante. On le voit notamment dans les liens entretenus, de

19. MEURISSE, Martin, Histoire de la naissance, du progrès et de la decadence de l'heresie dans la ville de Metz \& dans le pays Messin, Metz, Antoine, 1642, p. 553-554.

20. Vignal-Souleyreau, Marie-Catherine, Richelieu et la Lorraine, Paris, L'Harmattan, 2004.

21. BenoIST, Élie, Histoire de l'Édit de Nantes, Delft, Adrien Beman, 1693, t. 2, p. 138, 1695,

t. $3-1$, p. 538 et t. 3-3, p. 913-918.

22. LABRouSSE, Élisabeth, "Une foi, une loi, un roi? "La révocation de l'édit de Nantes, Genève - Paris, Labor et Fides - Payot, 1985; Daireaux, Luc, "Réduire les huguenots ». Protestants et pouvoirs en Normandie au XVII siècle, Paris, Honoré Champion, 2010.

23. LÉONARD, Julien, "Les pasteurs et la réunion des Églises au XVII ${ }^{\mathrm{e}}$ siècle : le cas de Paul Ferry ", Bulletin de la shpf, t. 156-1, 2010, p. 81-106.

24. VAlladier, André, Épitaphe panégyrique ou Le Pontife chrétien, Paris, Pierre Chevalier, 1612, p. 114.

25. BnF, NAF 22701, fol. 182. 
façon très discrète toutefois, avec des Églises établies à l'étranger, dans la possibilité d'envoyer les enfants étudier hors du royaume, mais aussi dans le nœud que constitue Metz au sein des réseaux d'échanges de convertis gênants, notamment des ecclésiastiques catholiques passés au protestantisme, en une époque où ceux-ci sont recherchés avec plus d'empressement par les autorités ${ }^{26}$.

Même si le processus de normalisation du statut des réformés de Metz par rapport à ceux du royaume ne suit pas un chemin linéaire et n'empêche pas des particularismes (par exemple le maintien jusqu'au bout hors des structures synodales, ou l'absence d'enregistrement de certaines déclarations royales), on peut tenter de dégager quelques moments importants. Il est révélateur que ceux-ci correspondent à des périodes de renforcement du pouvoir royal dans la ville. Prenons quelques exemples, loin d'être exhaustifs.

Le premier grand moment de la remise en cause de la tolérance accordée aux protestants de Metz se situe dès les années 1620, alors même qu'ils se tiennent à l'écart des " guerres de Rohan " qui aboutissent en 1629 au démantèlement du " parti huguenot " dans le royaume ${ }^{27}$. Envoyant au début de cette décennie des intendants pour rétablir l'ordre, la monarchie en profite pour désarmer les réformés messins lors de périodes de troubles, mais aussi pour installer, malgré leurs nombreuses remontrances parfois même relayées par le Magistrat, un collège de jésuites en 1622. Les enseignants qui y sont affectés sont également des controversistes, soutenus de surcroît par le pouvoir royal et ses relais en ville : cela se perçoit particulièrement bien en 1642, lorsque Louis XIII et le gouverneur dévot Schomberg autorisent le transfert de la propriété de l'ancien temple de la rue de la Chèvre aux jésuites, pour le collège, suscitant des procès sans fin qui sont pris en main après 1644 par Schomberg ${ }^{28}$. Les compensations promises ne sont pas réellement payées, mais le pouvoir promet de continuer à protéger les protestants. Malgré les garanties qui leur sont données à chaque étape de cette installation en forme de défi, les réformés de Metz sont clairement fragilisés, d'autant que les jésuites, à partir de 1657, prennent l'habitude de venir entendre les sermons au temple et d'en rapporter à la justice royale les éléments qu'ils considèrent contraires au régime de tolérance ${ }^{29}$.

C'est qu'entre 1622 et 1657 s'est produit un événement majeur dans la progression de la monarchie à Metz. En effet, en 1633 et 1641 ont été installés respectivement un parlement et un bailliage, mettant fin à une partie du particularisme municipal en introduisant très explicitement la souveraineté judiciaire du roi de France sur la cité, le Pays messin et l'évêché.

26. BoIsSON, Didier, Consciences en liberté? Itinéraires d'ecclésiastiques convertis au protestantisme (1631-1760), Paris, Honoré Champion, 2009.

27. Hubac, Jean, La paix d'Alès. La fin du parti huguenot? (27 juin 1629), Paris, Les Éditions de Paris/Max Chaleil, 2010.

28. MAZAuric, Roger, "Le Temple de Crèvecour ", Bulletin de la SHPF, t. 88-1, 1939, p. 5-21.

29. Nombreux mémoires aux Arch. dép. de la Moselle, D 11. 
Or, très rapidement, ces tribunaux consacrent une partie de leurs efforts à exercer certaines vexations à l'égard des réformés et à accorder une oreille complaisante à des dénonciations portées par les milieux dévots, notamment dans le clergé de Metz, instaurant une espèce de judiciarisation de la controverse confessionnelle. Cette évolution est défavorable au maintien du régime de tolérance, peu à peu vidé de sa substance par l'accumulation de décisions formant une jurisprudence hostile ${ }^{30}$. À cela s'ajoute le rôle personnel des gouverneurs, commandants et intendants qui se succèdent à Metz et qui tranchent la plupart des affaires dans un sens favorable aux catholiques. C'est ainsi que, malgré une longue procédure portant sur la validité d'un bail emphytéotique qui est finalement cassé sans grand fondement juridique, le temple de Chambière, construit au début du régime de tolérance initié en 1597, est ôté en 1663 aux réformés, car bâti sur un terrain appartenant aux bénédictins de l'abbaye de Saint-Vincent. L'Église réformée obtient un terrain dans le Retranchement de Guise pour bâtir un nouvel édifice mais la situation se dégrade rapidement, puisque le nouveau terrain, proche de celui occupé en 1561-1569, a été militarisé et catholicisé au cours des décennies précédentes, mettant de facto les réformés en position de communauté tolérée, mais isolée. Lorsqu'en 1666 Bossuet se prévaut du soutien des autorités pour mener avec le pasteur Paul Ferry un dialogue que l'historiographie qualifie d'" irénique ", c'est déjà en grande partie anachronique ${ }^{31}$.

Les mesures suivent désormais la chronologie des vexations que l'on retrouve ailleurs dans le royaume. Le pic suivant est donc atteint dès le début des années 1680, avec l'interdiction du temple champêtre de La Horgne, la fin de l'accès à certains honneurs ou professions, des mesures discriminatoires, stigmatisantes, ou symboliquement violentes, comme la lecture de l'Avertissement pastoral au consistoire en 1683, alors même que l'Église réformée de Metz n'est toujours pas une des Églises réformées de France et que le diocèse de Metz ne relève pas des assemblées du clergé ${ }^{32}$. Les autorités font alors face à une communauté encore puissamment structurée, mais dépassée par la violence des attaques. Même si certaines déclarations ne sont enregistrées au parlement de Metz que pour le ressort présidial de Sedan, le climat est délétère, et la tolérance accordée aux réformés messins ne tient qu'à un fil. L'expérience en est faite avec l'épisode de la révocation de l'édit de Nantes en $1685^{33}$. Aussitôt la nouvelle arrivée à

30. Villemin, Martial, Le Parlement de Metz et les protestants (de 1633 à 1735), thèse en histoire, dir. WAHL, Alfred, université de Metz, 1997.

31. LÉONARD, Julien, Etre pasteur au XVII siècle. Le ministère de Paul Ferry à Metz, Rennes, PUR, 2015, p. 179-182.

32. ANCILLON, Joseph, Recueil journalier de ce qui s'est passé de plus mémorable dans la Cité de Metz, pays Messin et aux environs (1656-1684), éd. CHABERT, François-Michel, Metz/ Paris, Rousseau-Pallez/Auguste Aubry, 2 vol., 1860-1866.

33. Pernot, Michel, "La révocation de l'édit de Nantes à Metz et dans le Pays messin ", dans Le Molgne, François-Yves et Michaux, Gérard (éd.), Protestants messins et mosellans..., op. cit., p. 123-145. 
Metz, le samedi 20 octobre, les réformés décident d'envoyer une députation vers le roi pour faire valoir ce qu'ils pensent être leurs privilèges. Même si les deux envoyés, dont l'ancien Charles Ancillon, le fils du pasteur David, ne peuvent partir que le lundi 22, après l'inventaire du temple et le début de sa destruction, les cahiers qu'ils emportent sont pleins de l'espoir de bénéficier des clauses des traités de Westphalie, comme les luthériens alsaciens ${ }^{34}$. Mais, précisément, ces protestants-là ne sont pas réformés, ni francophones, ce qui suffit à les rendre plus isolés et moins dangereux aux yeux du pouvoir. Les récits de cette députation nous montrent bien que les calvinistes messins sont désormais exactement traités comme les autres et qu'aucun particularisme n'est plus reconnu ${ }^{35}$, même si le simple fait d'avoir été reçus en cour est révélateur de ce qu'a été leur originalité. Les députés ne sont sans doute pas dupes toutefois, car leurs cahiers envisageaient plusieurs hypothèses, dont celle que la révocation serait appliquée sans ménagement à Metz, mais ils essaient de faire feu de tout bois en insistant sur les originalités de la ville, dont la présence juive. Pourtant, cette dernière était dénoncée par tous les chrétiens au XVII ${ }^{\mathrm{e}}$ siècle, y compris les réformés, qui cherchaient d'ailleurs souvent à se défendre de l'instrumentalisation, par les catholiques, des rares conversions de juifs pour controverser en réalité avec les protestants ${ }^{36}$. Mais les députés de l'Église réformée espéraient peut-être que l'argument pourrait être entendu, précisément parce que la tolérance accordée aux juifs à Metz était moins le fruit d'un rapport de force que de la seule bonne volonté royale.

\section{Une tolérance imposée : les juifs, instruments de la francisation?}

Les conditions d'installation des juifs à Metz expliquent largement qu'ils soient considérés comme des agents de développement de l'autorité royale. Comme le résumait l'historien Bernhard Blumenkranz, " ce ne sont [...] pas des Juifs qui seraient venus en France, c'est bien plutôt [...] la France qui est venue vers eux ${ }^{37}$ ". C'est que la monarchie a directement besoin d'eux et ce n'est en aucun cas un hasard si c'est le maréchal de Vieilleville qui instaure le régime de tolérance de fait dans les années 1560 : il est en effet le grand promoteur de la construction de la citadelle. Or, financer la présence de la garnison est un exercice difficile, la monarchie est toujours sur le fil du rasoir dans ce domaine, et doit sans cesse trouver des expédients. Il s'agit même d'un des enjeux majeurs de la consolidation de la présence

34. Bibliothèque du Protestantisme français, ms. 7652, pièce 108 et BnF, NAF 22703, fol. 52-53.

35. McKenna, Antony (et al., éd.), Correspondance de Pierre Bayle, Oxford, Voltaire Foundation, t. 7, 2009, p. 289-290 (lettre 675, écrite par Charles Ancillon, de Berlin le $8 / 18$ décembre 1686$)$.

36. LÉONARD, Julien, "Récits de conversion de juifs à Metz au XVII ${ }^{\mathrm{e}}$ siècle ", dans MARTIN, Philippe (dir.), Ephemera catholiques. L'imprimé au service de la religion (XVIXXI siècles), Paris, Beauchesne, 2012, p. 179-205.

37. Blumenkranz, Bernhard, « Les Juifs en Lorraine », Annales de l'Est, nº 3, 1967, p. 204. 
française en ville ${ }^{38}$. Les juifs qui s'installent à Metz, par les réseaux qu'ils entretiennent avec leurs coreligionnaires installés dans l'Empire proche, permettent de participer à leur manière à l'entretien de la garnison, notamment par le crédit à court terme, la fourniture de chevaux et de fourrage, devenant quasiment des auxiliaires de l'armée stationnée ${ }^{39}$. Cela ne va d'ailleurs pas sans heurts, et la population chrétienne comprend mal que les juifs ne soient pas plus systématiquement mis à contribution pour le logement des troupes : en 1646 et 1647, les instances municipales, composées de catholiques et de réformés, se plaignent au gouverneur Schomberg de ce que les juifs " jouissent de plus grands privilèges que les Chrétiens ", et qu'ils "n'ont logé ny fait aucune fourniture depuis longtemps, et ont profité au passage des gens de guerre ${ }^{40}$ ".

Dans ces conditions, on comprend toutefois mieux la tolérance que montre le pouvoir royal à leur égard. Alors que les Messins se plaignent régulièrement de leur progression numérique, réelle d'ailleurs, surtout dans les deux quarts extrêmes du XVII ${ }^{\mathrm{e}}$ siècle (de 120 à 398 individus de 1603 à 1621 et de 665 à 1080 de 1674 à 1698), les juifs voient les contours de l'autorisation d'installation se préciser dans les actes officiels les concernant, avec 58 ménages en 1614, 76 en 1624 et 96 en $1657^{41}$. Le pouvoir royal donne ainsi des signes clairs de sa protection, en partie pour montrer sa volonté aux chrétiens locaux hostiles. On l'a déjà vu en 1614, par le règlement qu'Épernon accorde pour leur établissement dans le quartier SaintFerroy, au risque de sa judaïsation. Mais on peut le voir aussi lorsque, malgré l'ironie des élites messines, un représentant de la communauté juive est accepté pour porter un compliment aux commissaires du roi chargés d'installer le parlement en août 1633, fixant le statut des juifs comme un corps juridiquement reconnu ${ }^{42}$.

C'est en 1657 que cette situation se fixe définitivement, lors du passage à Metz du roi Louis XIV, de Mazarin et de la reine mère Anne d'Autriche. Cette visite est symbolique du destin croisé des deux cultes tolérés. Alors que les autorités refusent de recevoir le compliment prévu par les députés du consistoire, que Louis XIII et Richelieu avaient pourtant accepté en $1631^{43}$, la famille royale va visiter la synagogue pour la fête de Soukkot, puis reçoit les dignitaires de la communauté, comme le rapporte le grand rabbin

38. GANTELET, Martial, L'absolutisme au miroir de la guerre. Le roi et Metz (1552-1661), Rennes, PUR, 2012.

39. Roos, Gilbert, Relations entre le gouvernement royal et les juifs du nord-est de la France au XVII siècle, Paris, Honoré Champion, 2000.

40. Arch. mun. de Metz, BB 45, fol. 75-77, 8 novembre 1646, et BB 48, fol. 70-71, 21 octobre 1647.

41. Meyer, Pierre-André, La communauté juive..., op. cit., p. 28.

42. BnF, Dupuy 498, fol. 218-221, lettre de Paul Joly à Pierre Dupuy, août 1633.

43. LÉONARD, Julien, " Les harangues de Paul Ferry, ou la prise de parole politique d'un pasteur réformé à Metz sous le régime de l'édit de Nantes ", dans Simiz, Stefano (éd.), La parole publique en ville des Réformes à la Révolution, Villeneuve d'Ascq, Presses universitaires du Septentrion, 2012, p. 85-103. 
Moïse Cohen $\mathrm{Narol}^{44}$. Ce dernier était arrivé en 1649 sans autorisation du gouverneur, comme c'était exigé depuis 1627 : mais le roi décide de lui pardonner, et d'accorder aux juifs des patentes de confirmation des privilèges, justifiant $a$ posteriori les nouvelles installations et permettant à certains notables de la communauté de ne pas porter les vêtements discriminants ${ }^{45}$. Ces faveurs sont toutefois ponctuelles, et le regard des agents du pouvoir royal partage finalement certains stéréotypes hostiles aux juifs. En 1699, dans le mémoire sur sa généralité de l'intendant Jacques-Étienne Turgot, les développements sur « la couleur de leur teint, leur figure et malpropreté, leur barbe, leur habillement Allemand " sont très révélateurs ${ }^{46}$. L'affaire Raphaël Lévy, du nom de ce juif de Boulay, en Lorraine ducale, accusé de meurtre rituel sur un enfant chrétien et condamné à mort par un arrêt du parlement du 16 janvier 1670, montre peut-être encore plus combien les préjugés antijudaïques, voire antisémites, sont ancrés jusque chez les représentants du roi ${ }^{47}$. La réhabilitation de Lévy par Louis XIV intervient quelques semaines plus tard à peine, et le roi tance les juifs de Metz pour ne pas l'avoir sollicité plus tôt, notamment parce que l'accusé n'était pas membre de la communauté messine. Mais le souverain lui-même partage la vision des juifs de ses contemporains, par exemple dans son édit de juin 1669 sur les intérêts autorisés, précisant que " les Juifs, établis en ladite ville de Metz, épuisent \& consomment nos sujets par d'autres usures encore plus excessives ${ }^{48}$ ".

Ce n'est qu'au début du XvIII ${ }^{\mathrm{e}}$ siècle, puis progressivement jusqu'à la Révolution que les juifs de Metz rentrent à leur tour dans le rang, désormais moins privilégiés économiquement à cause de la terrible taxe Brancas qui pèse sur eux depuis 1715, mais aussi moins isolés culturellement avec l'intégration, voire l'assimilation, d'une frange de la communauté ${ }^{49}$. Mais, à ce moment-là, il n'y a officiellement plus d'autre culte toléré depuis plusieurs décennies déjà.

Que retenir de ces destins communautaires croisés de deux cultes tolérés, certes dans des conditions très différentes, mais qui amènent la monarchie à tenter de se renforcer en ville au prix de deux politiques radicalement opposées? Tout d'abord, soulignons que le pragmatisme est le maître mot de l'attitude du pouvoir royal en ville, au moins jusqu'à l'installation du parlement en 1633, laissant donc certains privilèges s'enraciner. Dans les décennies suivantes, la francisation de Metz conduit le pouvoir à

44. CAHEN, Abraham, Le rabbinat de Metz..., op. cit., p. 32-33.

45. Emmery, Jean-Louis Claude (éd.), Recueil des Édits..., op. cit., t. 2, 1776, p. 565-572, et t. 4, Veuve Antoine \& Fils, 1787, p. 131-132.

46. Arch. dép. de la Moselle, J 6980, copie, p. 99.

47. BiRnBAUM, Pierre, Un récit de " meurtre rituel » au Grand Siècle. L'affaire Raphaël Lévy. Metz, 1669, Paris, Fayard, 2008.

48. Emmery, Jean-Louis Claude (éd.), Recueil des Édits..., op. cit., t. 5, p. 367.

49. Berkovitz, Jay R., "Acculturation and integration in eighteenth-century Metz ", Jewish History, t. 24-3, 2010, p. 271-294. 
remettre en cause le régime de tolérance accordé aux réformés, notamment en leur faisant subir le sort de leurs coreligionnaires du reste du royaume, ce qui n'allait pas de soi pour eux, tout en consolidant celui accordé aux juifs.

Comment comprendre, donc, que Louis XIV, grand pourfendeur des réformés jusque dans Metz, se soit accommodé de la présence des juifs? Plusieurs éléments de réponse, sans prétendre à l'exhaustivité, peuvent être avancés à titre d'hypothèses. Celle de l'Ersatzbürgertum (ou d'une notion dont il faudrait préciser les contours sémantiques), reprise par Stephan Laux ${ }^{50}$, est séduisante : la monarchie aurait accepté la tolérance vis-à-vis des juifs pour compenser les effets négatifs de la persécution des protestants et de leurs départs, nombreux dès les années 1680, et, comme le soulignait Patricia Behre Miskimin, la politique tant à l'égard des calvinistes que des juifs, malgré leurs contradictions apparentes, renforce la monarchie à Metz, même si sans doute l'État a été moins absolu qu'il n'y paraît dans son modèle ${ }^{51}$. Comme le souligne Stephan Laux, la monarchie d'Ancien Régime, en France ou dans le Brandebourg, est soucieuse des privilèges et des situations locales, ce qui explique certains particularismes que l'on prend trop souvent, de façon anachronique, pour des contradictions.

Cette tolérance à géométrie variable tient donc compte des intérêts du souverain, à la fois à l'échelle locale et à l'échelle du royaume, et l'on peut se risquer à d'autres réflexions. Sans doute les juifs ne constituent-ils pas le même danger que les réformés pour le modèle politico-religieux de Louis XIV, soutenu par son clergé. En effet, les chrétiens ne se convertissent pas au judaïsme à Metz, les spécificités culturelles sont telles que des barrières bien étanches sont établies. Au contraire, le calvinisme peut passer pour une concurrence d'autant plus grave pour des catholiques qu'elle n'implique pas les mêmes barrières. On peut aussi comprendre le maintien de la tolérance vis-à-vis des juifs de Metz par leur spécificité à l'échelle française et donc penser que si les réformés de la ville sont soumis au sort commun de leurs coreligionnaires, c'est que, précisément, ils leur ressemblent trop pour que cela justifie une exception. En ce sens, on pourrait se risquer à expliquer le maintien de la tolérance envers les juifs par la comparaison avec les luthériens d'Alsace par exemple. Leur faiblesse numérique et leur isolement ne remettent pas en cause le modèle de construction de l'État, mais peut même justifier leur maintien. Paradoxalement - en apparence du moins -, ces juifs, qui entretiennent des réseaux avec l'étranger et qui utilisent un yiddish incompréhensible pour les agents du pouvoir, semblent donc moins subversifs que des réformés pourtant habitués à l'absolutisme et aux grandes protestations de fidélité ${ }^{52}$.

50. LauX, Stephan, «"Ersatzbürgertum”... », art. cité.

51. BeHRe Miskimin, Patricia, One King, One Law, Three Faiths..., op. cit.

52. LABRousSE, Élisabeth, « La doctrine politique des huguenots, 1630-1685 ", Études théologiques et religieuses, t. 47-4, 1972, p. 421-429. 
Les juifs se maintiennent donc au XVIII ${ }^{\mathrm{e}}$ siècle, contrairement aux protestants, en partie parce qu'il s'agit d'un corps certes privilégié, mais facile à contrôler, et qui permet de renforcer la présence militaire royale, élément majeur de la francisation et de la normalisation de la ville; une fois cette normalisation acquise, le particularisme juif sera regardé avec plus de suspicion par la monarchie, malgré les progrès (ou à cause d'eux?) de l'acculturation.

\section{RÉSUMÉ}

La ville de Metz (20000 habitants), sous protection française depuis 1552, puis en voie de francisation politique jusqu'à l'annexion officielle en 1648 , constitue un laboratoire à part pour observer la coexistence confessionnelle et la tolérance accordée par la monarchie au XVII ${ }^{\mathrm{e}}$ siècle. On y voit la présence d'une forte minorité réformée (entre la moitié et le cinquième de la population) qui bénéficie de certaines libertés inconnues sous le régime de l'édit de Nantes, et d'une petite minorité juive, protégée par la monarchie malgré le renouvellement de l'édit d'expulsion du royaume en 1615 .

La tolérance accordée à ces deux communautés par le pouvoir politique est à géométrie variable. Tandis que les réformés connaissent un sort de moins en moins favorable de la part des autorités et du clergé catholique, jusqu'à devoir connaître les effets de la révocation de l'édit de Nantes qui supprime leur liberté de culte (1685), les juifs continuent à être protégés, malgré l'hostilité des chrétiens locaux. Il faut y voir une volonté très pragmatique de ne tenir compte que des intérêts (géo)-politiques et économiques les plus immédiats de la monarchie, aux échelles locale et nationale, dans une ville de frontière hautement stratégique.

\section{ABSTRACT}

The city of Metz (20,000 inhabitants), under French protection since 1552, then politically frenchified until the official annexation in 1648, can be viewed as a particular laboratory of confessional coexistence and of the tolerance granted by the monarchy during the $17^{\text {th }}$ century. We can see the presence of a strong Calvinist minority (between half and one-fifth of the population) enjoying a better status than that offered by the edict of Nantes, and of a small Jewish minority, under the protection of the monarchy despite the renewal of the expulsion edict in 1615.

The toleration granted to these two communities by the political power presents varying scales. While the Reformed lived under an increasingly uncomfortable regime, culminating with the revocation of the edict of Nantes that denied them freedom of worship (1685), the Jews kept on being protected, despite the hostility of local Christians. This was the result of the monarchy's pragmatic vision of the situation that only took into account its imperative (geo)political and economic interests, on local and national level, in a highly strategic frontier city. 